\title{
Models of nanoparticle transport in dielectrophoretic microdevices: prediction, parameter estimation and other applications
}

\begin{abstract}
This paper describes the applications of Fourier Bessel series models for characterising the transport of nanoparticles driven by dielectrophoretic forces and randomized by Brownian motion. Nanoparticle transport using dielectrophoresis continues to be an active area of research and models are fundamental for characterising the process. The models have very useful capabilities including prediction of nanoparticle transport, estimation of parameter values from experimental data, and data decomposition into space and time components. The models also give a frequency domain representation that will be applicable for time modulated dielectrophoretic nanoparticle transport.
\end{abstract}

Keyword: Dielectrophoresis; Fokker Planck equation; Fourier-Bessel series; Nanoparticle transport 